\title{
Selbstwertgefühl und ärgerbezogenes Verhalten
}

Self-Esteem and Anger-Related Reactions

\section{Manja Vollmann, Hannelore Weber und Monika Wiedig}

Institut für Psychologie, Universität Greifswald

Zusammenfassung: In der vorliegenden Studie mit $N=136$ studentischen Probanden wurde der Zusammenhang zwischen zwei Aspekten des Selbstkonzeptes, dem globalen Selbstwertgefühl und der Standfestigkeit gegenüber signifikanten anderen, und der Reaktion auf eine experimentell induzierte ärgerauslösende Provokation untersucht. Das Selbstkonzept sowie das habituelle ärgerbezogene Verhalten wurden eine Woche vor der experimentellen Provokation erfasst. Ärger wurde induziert, indem die Probanden in ungerechtfertigter Weise für einen von ihnen nicht verschuldeten Computerabsturz verantwortlich gemacht wurden. Im Anschluss an die Ärgerinduktion wurden über Fragebogen die emotionalen Reaktionen, das ärgerbezogene Verhalten, die Verhaltensziele und die Bewertung des Verhaltens erfasst. Die Ergebnisse zeigen, dass auf der habituellen Ebene hohe Ausprägungen des Selbstwertgefühls und der Standfestigkeit mit einer niedrigeren Neigung zu Anger-in verbunden waren. Das Selbstwertgefühl hatte auf das selbsteingeschätzte Verhalten und die Verhaltensziele in der konkreten Ärgersituation hingegen keinen Einfluss; eine höhere Standfestigkeit war jedoch mit weniger submissivem und ruminativem Verhalten sowie weniger Konfliktvermeidung und mehr Durchsetzungswillen verbunden. Die Ergebnisse werden im Hinblick auf den in der Literatur umstrittenen Zusammenhang zwischen Selbstwertgefühl, Ärgerneigung und Aggression diskutiert.

Schlüsselwörter: Selbstwertgefühl, Standfestigkeit, Ärger, Ärgerregulation, Aggression

Summary: In the present study with $N=136$ students the relation between two aspects of self-concept, i.e., global self-esteem and firmness facing significant others, and the behaviour in an experimental anger-provoking situation was examined. The self-concept and habitual anger behaviour were measured one week before the experimental provocation. To provoke anger, participants were blamed for a computer crash which in fact was not their fault. After the anger induction, emotional reactions, anger-related reactions, goals, and evaluations of the behaviour were assessed using questionnaires. The results show that regarding to habitual anger behaviour high self-esteem and firmness were related to a lower proneness towards anger-in. In the actual anger-provoking situation self-esteem was associated neither with self-reported anger-related reactions nor goals. However, high firmness was related to less submissive and ruminative behaviour as well as a lower intention to avoid conflicts and a higher intention to assert personal standards. The results are discussed with regard to the controversial relation between self-esteem, anger, and aggression.

Keywords: Self-esteem, anger, anger regulation, aggression 
Ärger ist eine im Alltag vergleichsweise häufig erlebte Emotion, die in der überwiegenden Mehrzahl aller Fälle in der Interaktion mit anderen Menschen erfahren wird (z. B. Averill, 1982; Scherer \& Tannenbaum, 1986). Kognitiven Emotionstheorien zufolge wird Ärger durch die Wahrnehmung ausgelöst, dass eine andere Person willentlich oder fahrlässig einen materiellen oder immateriellen Schaden verursacht und dabei mit ihrem Verhalten gegen bestehende Regeln verstößt (Averill, 1982; Ortony, Clore \& Collins, 1988; Smith \& Lazarus, 1993). Während mit diesen Annahmen die Art des erlittenen Schadens nicht weiter spezifiziert wird, vertritt Lazarus (Postscript zu Smith \& Lazarus, 1993) die These, dass Ärger nur dann entsteht, wenn mit dem Fehlverhalten eine Erniedrigung verbunden ist. Empirisch bestätigt sich, dass Angriffe auf Selbstwert und Selbstkonzept, beispielsweise ungerechtfertigte Kritik, Beleidigungen oder Hänseleien, zu den wichtigen ärgerauslösenden Faktoren zählen (Averill, 1982; Weber, 1994).

Wird Ärger durch selbstwertrelevante Angriffe ausgelöst, liegt es nahe zu vermuten, dass das Selbstwertgefühl einer Person ihre Reaktion auf einen solchen Angriff beeinflusst. In einer Reihe von Studien wurde daher der Zusammenhang zwischen Selbstwertgefühl, Ärgerneigung und ärgerbezogenem Verhalten untersucht. Zudem liegen Arbeiten vor, die unabhängig von der Entstehung von Ärger den Zusammenhang zwischen Selbstwertangriffen und Aggression überprüfen. Die Ergebnisse sind jedoch inkonsistent und widersprüchlich.

So deuten einige Befunde darauf hin, dass Personen mit einem niedrigen Selbstwertgefühl sich durch Frustrationen und Provokationen stärker in ihrem Selbstwert beeinträchtigt fühlen (Schütz, 1998, 2000) und auf verbale Angriffe mit mehr Ärger und Aggression reagieren als Personen mit einem höheren Selbstwertgefühl (zsf. Murray, 1985; Papps \& O'Carroll, 1998; siehe auch Nunn \& Thomas, 1999). Erklärt werden können diese Befunde durch die Annahme, dass ein niedriges Selbstwertgefühl die Vulnerabilität gegenüber Provokationen erhöht, weil die Verletzung eines ohnehin schon niedrigen Selbstwertgefühls als äußerst aversiv erlebt wird (vgl. Averill, 1982; Kernis, Granneman \& Barcley, 1989; Überblick Baumeister, Smart \& Boden, 1996; Papps \& O'Carroll, 1998). Ärger und Aggression, so die
Annahme, werden als Strategien eingesetzt, um in Form von Vergeltung das verletzte Selbstwertgefühl wiederherzustellen bzw. um durch aggressive Dominierung anderer die Selbstachtung zu erhöhen. Von Personen mit einem hohen Selbstwertgefühl wird hingegen angenommen, dass sie gewissermaßen immun sind gegenüber Kritik und Selbstwertbedrohung, da sie sich ihrer Selbstwertschätzung sicher sind (vgl. Baumeister et al., 1996).

Andere Studien legen das Gegenteil nahe, dass nämlich ein hohes Selbstwertgefühl zu mehr Ärger und Aggression führt. So gehen Baumeister und Koautoren (Baumeister \& Boden, 1998; Baumeister et al., 1996; Bushmann \& Baumeister, 1998; siehe auch Papps \& O'Carroll, 1998) davon aus, dass nicht ein niedriges, sondern ein unangemessen hohes Selbstwertgefühl zu vermehrtem Ärger und vor allem zu höherer Aggression führt, weil Angriffe angesichts des positiven Selbstbildes eher als ungerechtfertigt angesehen werden. Diesen Überlegungen zufolge macht insbesondere eine übertrieben positive Selbstsicht für Selbstwertangriffe vulnerabel, da Personen, die sich überschätzen, ständig Gefahr laufen, in ihrer Selbst(über)schätzung nicht bestätigt zu werden (Baumeister \& Boden, 1998).

\section{Ziele und Fragestellungen der vorliegenden Studie}

Die Annahme, dass ein (unangemessen) hohes Selbstwertgefühl den eigentlichen Risikofaktor für erhöhten Ärger und Aggression darstellt, ist eine Möglichkeit, durch eine Differenzierung des Konzeptes des Selbstwertgefühls die widersprüchlichen Ergebnisse zu erklären. Eine weitere Differenzierung wurde von Kernis et al. (1989; Kernis, 1993) vorgeschlagen. Diese Autoren postulieren, dass neben der Höhe auch die Stabilität des Selbstwertgefühls auf das Ärgererleben und Ärgerverhalten wirkt. Sie gehen davon aus, dass vornehmlich Personen mit einem hohen, aber instabilen Selbstwertgefühl zu Ärger und Aggression neigen, da sie aufgrund der situativen Schwankungen besonders sensibel gegenüber Fremdbewertungen sind. Aber auch bei einer Berücksichtigung dieser Aspekte des Selbstwertgefühls bleiben Fragen offen, die Gegenstand unserer Studie sind. 
Ein grundlegendes Problem in den vorliegenden Arbeiten ist die ungenügende Differenzierung der erfassten ärgerbezogenen Reaktionen. In vielen Studien steht das aggressive Verhalten im Vordergrund, während andere Formen der Reaktion, und hier vor allem konstruktive Reaktionen, kaum beachtet werden. Es ist aber anzunehmen, dass sich Personen in ihrer Reaktion auf einen Selbstwertangriff dadurch unterscheiden, dass selbstwerthohe Personen die Provokation offen und assertiv, dabei nicht aggressiv, zurückweisen, während selbstwertniedrige Personen sich ihr eher unterwerfen, den Ärger nicht zeigen, oder aber, wie vielfach vermutet wird, aggressiv reagieren, um den verletzten Selbstwert zu verteidigen (Schütz, 1998, 2000). Es ist daher eine erste Zielsetzung unserer Studie, die Hypothese zu überprüfen, dass sich ein höheres Selbstwertgefühl mit einer offen-konstruktiven Bewältigung einer selbstwertrelevanten Provokation verbindet, während ein niedrigeres Selbstwertgefühl mit einem submissiven und ruminativen Verhalten assoziiert ist. Zudem wird erwartet, dass Personen mit höherem Selbstwert in Übereinstimmung mit ihrer positiven Selbsteinschätzung ihr Verhalten als wirksamer und angemessener einschätzen und sich subjektiv damit wohler fühlen, als es Personen mit einem niedrigeren Selbstwertgefühl tun. Im Hinblick auf die Ärgerintensität bleibt angesichts der inkonsistenten Befunde offen, ob ein höheres Selbstwertgefühl mit geringerem oder höherem Ärger verbunden ist.

Ein zweites Ziel dieser Arbeit ist es, neben ärgerbezogenen Reaktionen auch die Ziele zu erfassen, die von provozierten Personen mit ihrer Reaktion auf eine Provokation verfolgt werden. Generell zählen Ziele in der Emotionsforschung zu den vernachlässigten Themen, obschon davon ausgegangen wird, dass die Emotionsregulation von Zielen geleitet wird (Gross, 1999; Laux \& Weber, 1991; Weber, 2004; Weber \& Titzmann, 2003). So ist zu erwarten, dass Personen mit einem niedrigen Selbstwertgefühl in der Reaktion auf eine Provokation vor allem die Ziele verfolgen, den verletzten Selbstwert zu schützen und zu verteidigen (Schütz, 1998). Selbstwerthohe Personen, so die Annahme, verfolgen hingegen stärker das assertive Ziel, den Provokateur in seine Grenzen zu weisen und ihn dazu zu bringen, sich künftig angemessen zu verhalten und Provokationen zu unterlassen.

Ein drittes Ziel unserer Untersuchung liegt in einer differenzierten Erfassung des Selbstwertgefühls. Vorliegende Arbeiten konzentrieren sich in der Regel auf den Einfluss des globalen Selbstwertgefühls. Wir erwarten hingegen, dass ein für den Umgang mit sozialen Konfliktsituationen besonders relevanter, spezifischer Aspekt des Selbstkonzeptes, nämlich die «Standfestigkeit gegenüber Gruppen und bedeutsamen anderen» (Deusinger, 1986) in einem engeren Zusammenhang mit ärgerbezogenem Verhalten steht als die allgemeine Selbstwertschätzung. Da wir selbstwertbezogene Unterschiede vor allem in der Assertivität erwarten, ist anzunehmen, dass die habituelle Selbsteinschätzung als standfest, das heißt als fähig, eigene Vorstellungen, Meinungen und Standpunkte gegenüber anderen, auch Autoritätspersonen, zu äußern und durchzusetzen, mit einem assertiven Verhalten in der Reaktion auf eine Provokation verbunden ist, während eine geringe Standfestigkeit mit submissivem und ruminativem Verhalten assoziiert ist. Mit dieser vergleichenden Erfassung von allgemeiner Selbstwertschätzung und spezifischer Kompetenzeinschätzung greifen wir die im Hinblick auf das globale Selbstwertgefühl postulierte Differenzierung zwischen einer auf dem zugeschriebenen Wert und einer auf der zugeschriebenen Kompetenz beruhenden Selbsteinschätzung auf (z. B. Tafarodi \& Swann, 2001; von Collani \& Herzberg, 2003).

Ärger ist in der Mehrzahl aller Episoden sozialer Natur und wird in sozialen Interaktionen ausgetragen. Um diesen für den Alltag typischen Kontext zumindest ansatzweise herzustellen, wird in unserer Studie Ärger durch das Verhalten eines Versuchsleiters bzw. einer Versuchsleiterin im Kontext eines Experimentes induziert. In Übereinstimmung mit der generellen Befundlage (Kring, 2000; Weber \& Piontek, 1995) erwarten wir im Hinblick auf das ärgerbezogene Verhalten von Frauen und Männern in dieser Situation keine bedeutsamen Unterschiede.

\section{Methode}

\section{Stichprobe}

An der Untersuchung nahmen 144 Studierende verschiedener Fächer der Universität Greifswald 
teil. Acht Personen wurden aufgrund der Informationen aus dem Nachgespräch von den Analysen ausgeschlossen (siehe Ablauf). Die verbleibende Stichprobe bestand aus 74 Frauen und 62 Männern $(M=23$ Jahre, $S D=3.4)$. Studierenden der Psychologie (40\% der Stichprobe) wurde für die Teilnahme Versuchspersonenzeit bescheinigt, und Studierende anderer Fachrichtungen erhielten eine Vergütung von 10 Euro.

\section{Ablauf}

Die Probanden wurden durch Aushänge im Institut für Psychologie und in der Mensa der Universität um Teilnahme und - bei Interesse - um eine Terminvereinbarung gebeten. Den Interessierten wurde mitgeteilt, dass das Thema der Untersuchung «Persönlichkeit und Konzentration» sei, und dass ihre Aufgabe darin liege, verschiedene Fragebogen sowie eine Konzentrationsaufgabe am Computer zu bearbeiten. Wurde das grundlegende Einverständnis zur Teilnahme gegeben, erfolgte die Datenerhebung zu zwei Zeitpunkten: Zunächst wurden den Probanden Fragebogen zur Erfassung von Persönlichkeitsmerkmalen mit der Bitte mitgegeben, diese zu dem für den zweiten Teil der Studie vereinbarten Termin mitzubringen. Zu diesem zweiten Zeitpunkt, in der Regel eine Woche später, erfolgte die experimentelle Induktion von Ärger im Kontext eines computerpräsentierten Stroop-Tests.

$\mathrm{Zu}$ Beginn der Laboruntersuchung wurde den Teilnehmern der Untertest «Buchstabenreihen» des Leistungsprüfsystems (LPS; Horn, 1983) vorgelegt, um die mitgeteilte Zielsetzung der Studie zu untermauern. Nach Abschluss des Tests bearbeiteten die Teilnehmer die Zustandsskala des State-Trait-Ärgerausdrucks-Inventars, deren Werte als Baseline für die nachfolgende Ärgerinduktion dienten. Die Ärgerinduktion erfolgte im Rahmen der Bearbeitung eines für diesen Zweck umprogrammierten Stroop-Tests (Stroop, 1935; Computerversion von Glaser, 1991). Bei diesem Test war es Aufgabe der Probanden, schnellstmöglich die Farbe eines auf dem Monitor eingeblendeten Farbwortes zu identifizieren und je nach Farbe des Wortes eine der drei farblich markierten Cursor-Tasten zu drücken. Den Probanden wurde mitgeteilt, dass die Richtigkeit der Antworten und die Reaktionszeiten erfasst wer- den. Nach vier Minuten funktionierender Testdurchführung wurde ein Programmabsturz simuliert, indem eine mit einem Warnton gekoppelte Fehlermeldung auf dem Bildschirm erschien. Daraufhin wurden die Probanden von dem Versuchsleiter - ungerechtfertigt - beschuldigt, trotz eindeutiger Instruktion zu Beginn der Testdurchführung eine falsche Taste, nämlich «enter», gedrückt zu haben (was durch die Anordnung der markierten Cursor-Tasten faktisch unmöglich war). Es wurde erklärt, dass aufgrund ihres Fehlers die Daten unbrauchbar seien, und sie daher die zugesagte Belohnung nicht erhalten würden. Im Anschluss an die Ärgerinduktion wurden den Probanden Fragebogen zur Erfassung des aktuellen emotionalen Befindens, der ärgerbezogenen Reaktionen und Ziele sowie der Bewertung des Verhaltens vorgelegt. Die Interaktion zwischen Proband und Versuchsleiter wurde durch eine Videokamera aufgenommen. Die Probanden wurden vor Beginn der Laboruntersuchung über die Videoaufnahme informiert.

Die Rolle der Versuchsleiter wurde von einer Studentin und einem Studenten der Psychologie, die kurz vor Abschluss des Studiums standen, übernommen. Es konnte davon ausgegangen werden, dass sie den jüngeren Studierenden der Psychologie und erst recht den Studierenden der anderen Fachrichtungen nicht bekannt waren. Beide Versuchsleiter wurden anhand von Videoaufnahmen sorgfältig in ihrem Provokationsverhalten trainiert und während der Untersuchung regelmäßig auf eine möglichst standardisierte Durchführung der Provokation überprüft. Weibliche Probanden wurden von der Versuchsleiterin, männliche Probanden von dem Versuchsleiter provoziert.

In einem Gespräch unmittelbar nach der Laboruntersuchung wurden die Probanden über die eigentliche Untersuchungsabsicht vollständig aufgeklärt und um ihr Einverständnis gebeten, die Videoaufnahmen für eine Verhaltensbeobachtung verwenden zu dürfen (siehe dazu Wiedig, 2004). Zudem wurden die Probanden gefragt, ob die Videokamera ihr Verhalten beeinflusst hat, sie vor der Teilnahme schon von dem Versuchsablauf gehört oder Zweifel an der wahren Zielsetzung des Experiments hatten. Acht Teilnehmer (4 Frauen, 4 Männer) wurden aufgrund dieser Befragung von der Datenanalyse ausgeschlossen. 


\section{Erhobene Variablen und Messinstrumente}

\section{Persönlichkeitsmerkmale}

Das allgemeine Selbstwertgefühl und das Selbstkonzept hinsichtlich der eigenen Standfestigkeit wurden mit der Frankfurter Selbstkonzeptskala zur allgemeinen Selbstwertschätzung (FSSW) und der Frankfurter Selbstkonzeptskala zur Standfestigkeit gegenüber Gruppen und bedeutsamen anderen (FSST) aus den Frankfurter Selbstkonzeptskalen (FSKN; Deusinger, 1986) erhoben. Beide Skalen erwiesen sich mit Konsistenzkoeffizienten von .93 (FSSW) und .90 (FSST) als hoch reliabel. Zur Erfassung der dispositionellen Ärgerneigung und des habituellen Ärgerausdrucks wurden die Trait-Anger-Skala sowie die drei Ärgerausdrucksskalen Anger-out, Anger-in und Anger Control des State-Trait-Ärgerausdrucks-Inventars (STAXI; Schwenkmezger, Hodapp \& Spielberger, 1992) eingesetzt. Die Reliabilität der vier STAXISkalen erwies sich als hoch (Cronbachs $\alpha$ s: TraitAnger: .84, Anger-out: .83, Anger-in: .78, Anger Control: .84).

\section{Emotionale Reaktionen}

Die Intensität des induzierten Ärgers wurde über die Differenz des Zustandsärgers vor und nach der Ärgerinduktion operationalisiert. Dazu wurde den Probanden zu beiden Zeitpunkten die State-Anger-Skala des STAXI (Schwenkmezger et al., 1992) vorgelegt. Die interne Konsistenz der Skala war bei beiden Messungen mit Cronbachs $\alpha$ s von .80 (vorher) und .90 (nachher) hoch. Das Ausmaß der erlebten Selbstwertverletzung wurde mit einer für diese Studie entwickelten Skala (Vollmann, 2001) erfasst, die den Probanden nach der Provokation vorgelegt wurde. Die acht Items dieser Skala beschreiben mögliche Gedanken und Gefühle nach einem Angriff auf den Selbstwert (z. B. «Ich war gekränkt»). Cronbachs $\alpha$ war mit .76 zufrieden stellend. Um eine Konzentration auf Ärger und Selbstwert zu vermeiden, wurde zusätzlich zu beiden Messzeitpunkten die Positive and Negative Affect Schedule (PANAS; Krohne, Egloff, Kohlmann \& Tausch, 1996) vorgegeben; auf diese Ergebnisse wird hier jedoch nicht eingegangen (siehe Wiedig, 2004).
Ärgerbezogene Reaktionen und Ziele

Das auf die Provokation bezogene Verhalten und die damit verfolgten Ziele wurden mit dem Fragebogen zu ärgerbezogenen Reaktionen und Zielen (AERZ; Weber \& Titzmann, 2003) erhoben. Der AERZ erfasst in sechs Subskalen mit je vier Items die Reaktionsformen Ausbruch, Brüten, Unterwerfung, Feedback, Distanzierung und Humor und ebenfalls in sechs Subskalen mit je vier Items folgende Ziele: Durchsetzung von allgemeinen Normen, Durchsetzung von persönlichen Standards, Vermeidung von Konflikten, Abschätzung von Kosten, Regulation von Affekt und Schutz des Ansehens. Da der AERZ in seiner Originalform (Weber \& Titzmann, 2003) habituelle Ärgerreaktionen und Verhaltensziele erfragt, wurden die Items für diese Untersuchung situationsbezogen umformuliert. Die Reliabilität der Skalen erwies sich mit Ausnahme von drei Skalen mit Werten von Cronbachs $\alpha<.70$ als zufrieden stellend (siehe Tabelle 1 ).

Tabelle 1. Korrelationen zwischen Selbstwertgefühl, Standfestigkeit und den ärgerbezogenen Reaktionen und Zielen.

\begin{tabular}{|c|c|c|c|}
\hline & $\begin{array}{l}\text { Cron- } \\
\text { bachs } \alpha\end{array}$ & $\begin{array}{l}\text { Selbst- } \\
\text { wert }^{\mathrm{a}}\end{array}$ & $\begin{array}{l}\text { Stand } \\
\text { festig- } \\
\text { keit }^{\mathrm{a}}\end{array}$ \\
\hline \multicolumn{4}{|c|}{ Ärgerbezogene Reaktionen } \\
\hline Brüten & .68 & -.10 & $-.30^{* * *}$ \\
\hline Unterwerfung & .76 & -.14 & $-.16^{(*)}$ \\
\hline Feedback & .78 & .01 & .08 \\
\hline Distanzierung & .58 & .00 & .02 \\
\hline Humor & .92 & .01 & $\begin{array}{c}.13 \\
-.12^{\mathrm{b}} \\
.26^{* \mathrm{c}}\end{array}$ \\
\hline \multicolumn{4}{|l|}{ Ärgerbezogene Ziele } \\
\hline $\begin{array}{l}\text { Durchsetzung } \\
\text { sozialer Normen }\end{array}$ & .86 & -.02 & .10 \\
\hline $\begin{array}{l}\text { Durchsetzung } \\
\text { persönlicher }\end{array}$ & .86 & $\begin{array}{l}-.04 \\
-.25^{* b}\end{array}$ & $.17^{*}$ \\
\hline Standards & & $.25^{* \mathrm{c}}$ & \\
\hline $\begin{array}{l}\text { Vermeidung } \\
\text { von Konflikten }\end{array}$ & .77 & -.15 & $-.26^{* *}$ \\
\hline $\begin{array}{l}\text { Abschätzung } \\
\text { von Kosten }\end{array}$ & .67 & .08 & .10 \\
\hline $\begin{array}{l}\text { Regulation } \\
\text { von Affekt }\end{array}$ & .72 & $\begin{array}{c}-.11 \\
.00^{\mathrm{b}} \\
-.19^{\mathrm{c}}\end{array}$ & -.08 \\
\hline $\begin{array}{l}\text { Schutz des } \\
\text { Ansehens }\end{array}$ & .75 & -.05 & -.12 \\
\hline
\end{tabular}

Anmerkungen: $N=136$. ${ }^{a}$ Ärgerintensität nach der Provokation ist auspartialisiert. ${ }^{b}$ nur Frauen $(n=74)$. ${ }^{c}$ nur Männer $(n$ =62). $\left.{ }^{*}\right) p=.06, * p<.05, * * p<.01, * * * p<.001$ (zweiseitig) 


\section{Selbstbewertung des Verhaltens}

Die Bewertung des in der Reaktion auf die Provokation gezeigten Verhaltens hinsichtlich seiner Wirksamkeit und Angemessenheit wurde über zwei bipolar angeordnete Adjektive (unwirksam - wirksam und unangemessen - angemessen) auf einer 7-stufigen Skala erfasst. Zudem wurde über eine 5-stufige Skala erfragt, wie zufrieden die Probanden mit ihrem Verhalten waren (sehr unzufrieden - sehr zufrieden) und wie wohl sie sich damit fühlten (sehr unwohl - sehr wohl). Da diese beiden Items hoch $(r=.77)$ miteinander korrelierten, wurden sie zu einer globalen Einschätzung der Zufriedenheit mit dem eigenen Verhalten zusammengefasst.

\section{Ergebnisse}

\section{Geschlechtsunterschiede}

Vor der Prüfung der Hypothesen wurden alle Variablen auf Geschlechtsunterschiede hin untersucht. Signifikante Unterschiede zwischen Männern und Frauen ergaben sich entgegen unserer Erwartung in mehreren Variablen: In der Gruppe der Männer waren Standfestigkeit, Anger Control, Humor, das Ziel der Durchsetzung eigener Standards sowie die selbsteingeschätzte Wirksamkeit und Zufriedenheit mit dem Verhalten höher ausgeprägt. Für die Gruppe der Frauen ergaben sich höhere Mittelwerte für Anger-out, erlebte Ärgerintensität und Selbstwertverletzung, Brüten sowie die Ziele Konfliktvermeidung und Affektregulation (Daten siehe Vollmann, 2001, und Wiedig, 2004). Angesichts dieser Unterschiede wurden zunächst alle Analysen getrennt für beide Geschlechter gerechnet und geprüft, inwieweit sich die Ergebnisse von Männern und Frauen signifikant voneinander unterscheiden. Dies war nur für insgesamt fünf Korrelationen der Fall; daher werden hier die Ergebnisse für die gesamte Stichprobe dargestellt und geschlechtsspezifische Zusammenhänge nur für die signifikant unterschiedlichen Ergebnisse berichtet.

\section{Wirksamkeit der Ärgerinduktion}

Der signifikante Anstieg des zu den beiden Messzeitpunkten vor und nach der Provokation erfassten Zustandsärgers zeigt, dass die gewünschte Ärgerinduktion gelungen ist; $M$ (vorher) $=11.31, M$ $($ nachher $)=13.99, t(135)=-6.98, p<.001, d=$ 0.85. Der Ärgeranstieg ist für die Gruppe der Frauen $(M=4.14)$ im Vergleich zur Gruppe der Männer $(M=.94)$ signifikant höher $(t(134)=4.43, \mathrm{p}<$ $.001)$; er ist aber in beiden Gruppen signifikant.

\section{Habituelles ärgerbezogenes Verhalten}

Hinsichtlich der dispositionellen Ärgerneigung ergab sich nur für die Männer $(r=-.44, p<.001)$, nicht aber für die Frauen $(r=-.06, n s)$, ein signifikant negativer Zusammenhang zum Selbstwertgefühl. Für die Standfestigkeit zeigte sich keine signifikante Beziehung zur Ärgerneigung $(r=-.07$, $n s)$. In Bezug auf das habituelle Ärgerverhalten wurden für Anger-in signifikant negative Korrelationen sowohl mit dem Selbstwertgefühl ( $r=-.39$, $p<.001)$ als auch mit der Standfestigkeit $(r=-.31$, $p<.001$ ) ermittelt. Für Anger-out und Anger Control ergaben sich keine signifikanten Zusammenhänge zum Selbstwertgefühl und zur Standfestigkeit (Korrelationen zwischen -.11 und .07, ns).

\section{Ärgerintensität und Selbstwertverletzung}

Weder für das Selbstwertgefühl $(r=.16, p=.07)$ noch für die Standfestigkeit $(r=.01, n s)$ ergaben sich signifikante Zusammenhänge zum Anstieg des Zustandsärgers nach der Provokation. Hingegen war, wie erwartet, der Selbstwert nach der Ärgerinduktion in der subjektiven Einschätzung umso stärker verletzt, je niedriger das Selbstwertgefühl $(r=-.27, p<.01)$ und die Standfestigkeit $(r=-.40, p<.001)$ waren.

\section{Ärgerbezogene Reaktionen und Ziele}

Tabelle 1 zeigt die Korrelationen zwischen Selbstwertgefühl, Standfestigkeit und den ärgerbezogenen Reaktionen sowie den Verhaltenszielen. Bei allen Korrelationen wurde der Einfluss der nach der Provokation erfassten Ärgerintensität kontrol- 
liert. Die Reaktions-Skala Ausbruch wurde von den weiteren Analysen ausgeschlossen, da dieses Verhalten von den Probanden in einem sehr geringen Ausmaß berichtet wurde $(M=4.34, S D=.90$, Wertebereich 4-16).

Entgegen unseren Hypothesen ergaben sich keine signifikanten Zusammenhänge zwischen dem Selbstwertgefühl und den selbstberichteten Reaktionen auf die Provokation. Hingegen zeigte sich, wie erwartet, dass eine niedrige Ausprägung in Standfestigkeit mit einer höheren Ausprägung in der gedanklichen Weiterbeschäftigung mit der Provokation (Brüten) sowie der Tendenz, eigene Interessen und Rechte aufzugeben, um offene Auseinandersetzungen zu vermeiden, assoziiert war. Zudem waren für die Männer, nicht jedoch für die Frauen, Standfestigkeit und Humor signifikant positiv verbunden.

In Bezug auf die Verhaltensziele ergab sich nur für das Verhaltensziel Durchsetzung persönlicher Standards eine signifikante, allerdings für Männer und Frauen entgegengesetzte, Beziehung zum Selbstwertgefühl. Während das Ziel, persönliche Standards durchzusetzen, bei Frauen mit einem höheren Selbstwertgefühl einherging, war es bei Männern mit einem niedrigeren Selbstwertgefühl assoziiert. Für die Standfestigkeit zeigte sich, wie erwartet, eine signifikant negative Korrelation mit dem Verhaltensziel Konfliktvermeidung und eine signifikant positive Beziehung zum Ziel, persönliche Standards durchzusetzen.

\section{Selbstbewertung des ärgerbezogenen Verhaltens}

Auch bei den Korrelationen zwischen Selbstwertgefühl, Standfestigkeit und der Selbstbewertung des Verhaltens wurde der Einfluss der Ärgerintensität nach der Provokation kontrolliert. Wie erwartet, wurde das eigene Verhalten als angemessener bewertet, je höher das Selbstwertgefühl ausgeprägt war $(r=.19, p<.05)$. Zudem war für die Gruppe der Frauen $(r=.33, p<.01)$, nicht aber der Männer $(r=-.10, n s)$, die Einschätzung des Verhaltens als wirksam mit einem höheren Selbstwert verbunden. Für die Standfestigkeit zeigte sich, dass das Verhalten als angemessener $(r=.28, p<$ $.01)$ und als wirksamer $(r=.25, p<.01)$ bewertet wurde, je höher die Standfestigkeit war. Die Zufriedenheit mit dem eigenen Verhalten war zudem stärker ausgeprägt, je höher das Selbstwertgefühl $(r=.30, p<.001)$ und die Standfestigkeit $(r=.28$, $p<.01)$ waren.

\section{Diskussion}

In der vorliegenden Studie wurde auf der Grundlage einer experimentell induzierten Provokation untersucht, in welcher Weise Unterschiede im Selbstwertgefühl und in der Selbsteinschätzung als «standfest» mit Unterschieden in der Ärgerneigung und dem ärgerbezogenen Verhalten verbunden sind.

Entgegen unserer Erwartung hatte das Selbstwertgefühl offenbar keinen Einfluss auf das in der Reaktion auf die experimentelle Provokation gezeigte Verhalten. Das gilt nicht nur für die hier berichtete Selbsteinschätzung der Studienteilnehmer, sondern auch für die Fremdeinschätzung ihres Verhaltens, die auf der Grundlage der Videoaufnahmen durch vier unabhängige Beobachter erfolgte, die zu ihrer Einschätzung eine entsprechende Variante des AERZ benutzten (Wiedig, 2004). Zusammenhänge zum Selbstwertgefühl ergaben sich hingegen in der Form, dass ein höheres Selbstwertgefühl einherging mit der Selbstbewertung des Verhaltens als angemessen und mit einer höheren Zufriedenheit mit dem eigenen Verhalten. Unterschiede zeigten sich zudem in der Einschätzung des habituellen Ärgerverhaltens. Ein höheres Selbstwertgefühl war mit einer geringeren Neigung zu Anger-in verbunden. Damit äußert sich das Selbstwertgefühl zwar in einer generalisierten positiveren bzw. negativeren Einschätzung des eigenen Verhaltens bzw. der eigenen Person, nicht jedoch in unterschiedlichen Reaktionen auf eine Provokation in einer konkreten Situation.

Im Unterschied zum Selbstwertgefühl zeigten sich die theoretisch erwarteten Zusammenhänge zwischen Selbstwert und ärgerbezogenem Verhalten für die spezifische, auf der Einschätzung von Kompetenzen beruhende Selbstkonzeptkomponente Standfestigkeit gegenüber signifikanten anderen. Eine höhere Standfestigkeit war verbunden mit einer geringeren Selbsteinschätzung des Verhaltens als unterwürfig und ruminativ sowie mit einer geringeren Neigung zur Konfliktvermeidung und einem höheren Durchsetzungswillen. Diese Selbsteinschätzung des aktuellen Verhaltens 
stimmt überein mit der für das habituelle Verhalten berichteten geringeren Neigung zu Anger-in. Auffallend ist jedoch, dass die Standfestigkeit entgegen unseren Annahmen nicht mit einem verstärkt assertiven Verhalten einhergeht, sondern lediglich mit einer Abschwächung des submissiven und ruminativen Verhaltens. Damit bleiben die Einflussfaktoren oder Korrelate des assertiven Verhaltens offen, obschon die Selbsteinschätzung sozialer Kompetenzen wie im Falle der Standfestigkeit eine theoretisch besonders erfolgversprechende Kandidatin darstellt (siehe auch Weber, Wiedig, Freyer \& Gralher, 2003).

Mit Blick auf die grundlegende Frage nach dem Zusammenhang zwischen Selbstwertgefühl, Ärgerneigung und Aggression legen die Ergebnisse nahe, dass zusätzlich zu den Differenzierungen, die bereits im Hinblick auf die Stabilität und Angemessenheit des Selbstwertgefühls postuliert werden (Baumeister et al., 1996; Kernis et al., 1989), auch eine inhaltliche Differenzierung des globalen Selbstwertgefühls (Tafarodi \& Swann, 2001) sinnvoll ist. Wenn überhaupt, wird unseren Ergebnissen zufolge die Reaktion auf eine Provokation zumindest in der Selbstwahrnehmung weniger durch den generellen Wert beeinflusst, den Personen sich selbst beimessen, sondern durch relevante Kompetenzen, die sie sich zuschreiben. Damit bestätigt sich einmal mehr der Stellenwert der Selbstwirksamkeit im Hinblick auf die Bewältigung kritischer Situationen (Bandura, 1997).

Der Zusammenhang zwischen Selbstwertgefühl und aggressivem Verhalten, der in der Literatur besondere Aufmerksamkeit findet (Baumeister et al., 1996), konnte in der vorliegenden Studie leider nicht überprüft werden, da das Ausmaß an aggressiven Reaktionen verschwindend gering war. Dies ist nicht überraschend, da die gewählte experimentelle Situation das Verhaltensspektrum gerade im Hinblick auf Aggression stark einschränkt. Andererseits ist die in experimentellen Designs häufig realisierte artifizielle Aufforderung zu - objektiv erfassbarem - aggressivem Verhalten ebenso unzureichend, da alle sozial-konstruktiven Verhaltensoptionen ausgeblendet bleiben. Es erscheint jedoch schwierig, ethisch vertretbare experimentelle Situationen zu schaffen, in denen ohne Vorgabe die ganze Bandbreite ärgerbezogenen Verhaltens gezeigt werden kann. Aber erst ein solches Design ließe empirisch fundierte Rückschlüsse auf die Zusammenhänge zwi- schen Selbstwertgefühl, Ärgerneigung und ärgerbezogenem Verhalten zu.

Für das Geschlecht zeigten sich für eine Reihe von Variablen Niveauunterschiede, vor allem für das Ausmaß an selbstberichteter Ärgerintensität, die für die Gruppe der Frauen deutlich höher war. Mit wenigen Ausnahmen ergaben sich jedoch in den geprüften Zusammenhängen zwischen Selbstwertgefühl, Standfestigkeit und ärgerbezogenem Verhalten keine signifikanten Unterschiede zwischen Männern und Frauen. Dabei fällt auf, dass für die Gruppe der Männer ein höheres Selbstwertgefühl mit einer geringeren habituellen Ärgerneigung sowie einer geringeren Ausprägung in den beiden Zielen Affektregulation und Durchsetzung eigener Standards verbunden war, insgesamt also mit einem mehr distanzierten und «abgeklärten» Verhalten. In der Gruppe der Frauen hingegen korrelierte ein höheres Selbstwertgefühl mit einer höheren Einschätzung der Wirksamkeit ihres gezeigten Verhaltens sowie einer höheren Ausprägung in dem Ziel, die eigenen Standards durchzusetzen. Damit scheint sich ein höheres Selbstwertgefühl mit einer den Geschlechtsstereotypen «aggressiver Mann» und «submissive Frau» gegenläufigen Selbsteinschätzung bei beiden Geschlechtern zu verbinden (Nunn \& Thomas, 1999).

Generell neigten die Frauen in der vorliegenden Studie dazu, sich im Vergleich zu den Männern als ärgerlicher, verletzter, unterwürfiger, ruminativer und unzufriedener zu beschreiben, also als insgesamt vulnerabler gegenüber Provokationen und weniger effektiv in ihrer Reaktion. Damit bestätigt sich in der Selbstdarstellung der Frauen zwar das Bild der weniger assertiven Frau (Überblick Kring, 2000; Weber \& Piontek, 1995), doch dies wird nur bedingt durch die Fremdeinschätzung gestützt, derzufolge die Frauen zwar als ruminativer eingeschätzt werden, ihnen aber auch ein höheres Ausmaß an Feedback zugeschrieben wird (Wiedig, 2004). Auch stehen die hier gefundenen Unterschiede Studienergebnissen entgegen, in denen im Hinblick auf ärgerbezogenes Verhalten keine oder nur inkonsistente Unterschiede gefunden wurden (Kring, 2000; Weber \& Piontek, 1995). Eine Schwäche der Studie liegt darin, dass nur geschlechtshomogene Dyaden untersucht wurden. In zukünftigen Studien sollte daher geprüft werden, welche Geschlechtsunterschiede sich bei einem vollständigen Untersuchungsdesign ergeben, bei dem eine Frau und ein Mann jeweils beide Ge- 
schlechter provozieren. Allerdings bleiben auch bei einem solchen erweiterten Design wie bereits in der vorliegenden Studie Geschlecht und Persönlichkeit des Provokateurs - unlösbar - konfundiert. Versuchsleitereffekte können lediglich durch den Vergleich unterschiedlicher Provokateure überprüft werden.

\section{Autorenhinweise}

Das methodische Vorgehen der Untersuchung steht im Einklang mit den Ethical Principles of Psychologists and Code of Conduct der APA (2002).

Teile der Studie wurden auf dem 43. Kongress der Deutschen Gesellschaft für Psychologie (DGPs) in Berlin, 22.-26.9.2002, sowie auf der 16th Conference of the European Health Psychology Society (EHPS) in Lissabon, 02.-05.10.2002, präsentiert.

\section{Literatur}

Averill, J.R. (1982). Anger and aggression. An essay on emotion. New York: Springer-Verlag.

Bandura, A. (1997). Self-efficacy: The exercise of control. New York: W.H. Freeman.

Baumeister, R.F. \& Boden, J.M. (1998). Aggression and the self: High self-esteem, low self-control, and ego threat. In R.G. Geen \& E. Donnerstein (Eds.), Human aggression: Theories, research, and implications for social policy (pp. 111-138). New York: Academic Press.

Baumeister, R.F., Smart, L. \& Boden, J.M. (1996). Relation of threatened egotism to violence and aggression: The dark side of high self-esteem. Psychological Review, 103, 5-33.

Bushman, B.J. \& Baumeister, R.F. (1998). Threatened egotism, narcissism, self-esteem, and direct and displaced aggression: Does self-love or self-hate lead to violence? Journal of Personality and Social Psychology, 75, 219-229.

Deusinger, I.M. (1986). Die Frankfurter Selbstkonzeptskalen. Göttingen: Hogrefe.

Glaser, W. (1991). Computerprogramm für Stroop. http://www.uni-tuebingen.de/psi/alteversion/soft/soft. htm.

Gross, J.J. (1999). Emotion regulation: Past, present, future. Cognition and Emotion, 13, 551-573.

Horn, W. (1983). Leistungsprüfsystem: LPS (2., erw. u. verb. Aufl.). Göttingen: Hogrefe.

Kernis, M.H. (1993). The roles of stability and level of self-esteem in psychological functioning. In R.F. Baumeister (Ed.), Self-esteem: The puzzle of low self-regard. Plenum series in social/clinical psychology (pp. 167-182). New York: Plenum.

Kernis, M.H., Grannemann, B.D. \& Barclay, L.C. (1989). Stability and level of self-esteem as predictors of anger arousal and hostility. Journal of Personality and Social Psychology, 56, 1013-1022.

Kring, A.M. (2000). Gender and anger. In A.H. Fischer (Ed.), Gender and emotion: Social psychological perspectives. (pp. 211-231). New York: Cambridge University Press.

Krohne, H.W., Egloff, B., Kohlmann, C.W. \& Tausch, A. (1996). Untersuchungen mit einer deutschen Version der «Positive and Negative Affect Schedule» (PANAS). Diagnostica, 42, 139-156.

Laux, L. \& Weber, H. (1991). Presentation of self in coping with anger and anxiety: An intentional approach. Anxiety Research, 3, 233-255.

Murray, E.J. (1985). Coping and anger. In T.M. Field, P.M. McCabe \& N. Schneiderman (Eds.), Stress and coping (pp. 243-262). Hillsdale, NJ: Erlbaum.

Nunn, J.S. \& Thomas, S.L. (1999). The angry male and the passive female: The role of gender and self-esteem in anger expression. Social Behavior and Personality, $27,145-154$.

Ortony, A., Clore, G.L. \& Collins, A. (1988). The cognitive structure of emotions. New York: Cambridge University Press.

Papps, B.P. \& O'Carroll, R.E. (1998). Extremes of self-esteem and narcissism and the experience and expression of anger and aggression. Aggressive Behavior, 24, 421438.

Scherer, K.R. \& Tannenbaum, P.H. (1986). Emotional experiences in everyday life: A survey approach. Motivation and Emotion, 10, 295-314.

Schütz, A. (1998). Coping with threats to self-esteem: the differing patterns of subjects with high versus low trait self-esteem in first-person accounts. European Journal of Personality, 12, 169-186.

Schütz, A. (2000). Psychologie des Selbstwertgefühls. Von Selbstakzeptanz bis Arroganz. Stuttgart: Kohlhammer.

Schwenkmezger, P., Hodapp, V. \& Spielberger, C.D. (1992). Das State-Trait-Ärgerausdrucks-Inventar (STAXI). Bern: Huber.

Smith, C.A. \& Lazarus, R.S. (1993). Appraisal components, core relational themes, and the emotions. Cognition and Emotion, 7, 233-269.

Stroop, R.J. (1935). Studies of interference in serial verbal reactions. Journal of Experimental Psychology, 18, 643-662.

Tafarodi, R.W. \& Swann, W.B. (2001). Two-dimensional self-esteem: Theory and measurement. Personality and Individual Differences, 31, 653-673.

Vollmann, M. (2001). Der Zusammenhang zwischen Selbstwertgefühl und Ärger. Unveröffentlichte Di- 
plomarbeit. Institut für Psychologie, Universität Greifswald.

von Collani, G. \& Herzberg, P.Y. (2003). Zur internen Struktur des globalen Selbstwertgefühls nach Rosenberg. Zeitschrift für Differentielle und Diagnostische Psychologie, 24, 9-22.

Weber, H. (1994). Ärger. Psychologie einer alläglichen Emotion. Weinheim: Juventa.

Weber, H. (2003, accepted). Explorations in the social construction of anger. Motivation and Emotion.

Weber, H. \& Piontek, R. (1995). Geschlechtsunterschiede in der Bewältigung von Ärger - ein Mythos? Zeitschrift für Gesundheitspsychologie, 3, 59-83.

Weber, H. \& Titzmann, P. (2003). Ärgerbezogene Reaktionen und Ziele: Entwicklung eines neuen Fragebogens. Diagnostica, 49, 97-109.

Weber, H., Wiedig, M., Freyer, J. \& Gralher, J. (1993).
Social anxiety and anger regulation. Manuscript submitted for publication.

Wiedig, M. (2004). Ärger und soziale Regeln. Ärgererleben und Ärgerverhalten in der Selbst- und Fremdwahrnehmung. Hamburg: Verlag Dr. Kovac.

Dipl.-Psych. Manja Vollmann

Universität Greifswald

Institut für Psychologie

Franz-Mehring-Str. 47

17491 Greifswald

Tel. +493834863773

Fax +493834863763

E-mailvollmann@uni-greifswald.de 